\title{
PERSISTENCE BARCODED VEHICULAR TRAFFIC VIDEOS. A TOPOLOGY OF DATA APPROACH TO SHAPE TRACKING
}

\author{
A.P.H. DON, J.F. PETERS, AND S. RAMANNA \\ Dedicated to Enrico Betti and Som Naimpally
}

\begin{abstract}
This paper introduces a computational CW topology of data approach to tracking the persistence of image object shapes that appear in triangulated video frames. Shapes are cell complexes are viewed in the context of an Alexandroff-Hopf-Whitehead CW (Closure finite Weak) topological space. Fermi energy and Betti numbers are used to construct persistence barcodes derived from nested cycles (optical vortexes) inherent in triangulated video frame shapes. An application of this approach is given in terms of Ghrist persistence barcoding of vehicular traffic videos.
\end{abstract}

\section{CONTENTS}

1. Introduction

2. Preliminaries

2.1. Betti numbers

2.2. Fermi energy

2.3. Time Complexity Analysis

3. Results

References

\section{INTRODUCTION}

This paper tackles the problem of tracking the persistence of moving shapes in a video by reducing each triangulated video frame to a collection of elementary cells. Each video frame is a finite, bounded region of the Euclidean plane. A cell in the Euclidean plane is either a 0-cell (vertex) or 1-cell (edge) or 2-cell (filled triangle). A cell complex is a collection cells attached to each other by edges or by having one or more common vertices.. A nonvoid collection of cell complexes $K$ has a Closure finite Weak (CW) topology, provided $K$ is Hausdorff (every pair of distinct

2010 Mathematics Subject Classification. 54C56 (Shape Theory); 55R40 (Homology of classifying shapes); $55 \mathrm{U} 10$ (Cell complexes).

Key words and phrases. Betti Number, Cell Complex, CW Topological Space, Optical Vortex Nerve, Persistence Barcode, Shape, Video Frame.

The research has been supported by the Natural Sciences \& Engineering Research Council of Canada (NSERC) discovery grants 185986 and 194376, Instituto Nazionale di Alta Matematica (INdAM) Francesco Severi, Gruppo Nazionale per le Strutture Algebriche, Geometriche e Loro Applicazioni grant 9920160 000362, n.prot U 2016/000036 and Scientific and Technological Research Council of Turkey (TÜBİTAK) Scientific Human Resources Development (BIDEB) under grant no: 2221-1059B211301223. 
cells is contained in disjoint neighbourhoods [12, §5.1, p. 94]) and the collection of cell complexes in $K$ satisfy the Alexandroff-Hopf-Whitehead [2, §III, starting on page 124], [22, pp. 315-317], [23, §5, p. 223] conditions, namely, containment (the closure of each cell complex is in $K$ ) and intersection (the nonempty intersection of cell complexes is in $K$ ). The focus in this work is on detecting nesting cycles in optical vortexes on the barycenters of triangles covering video frame shapes.

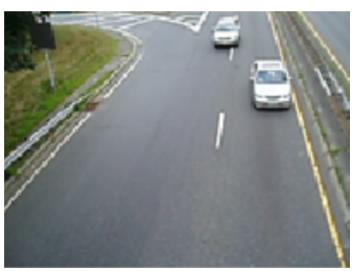

1.1: Traffic video frame

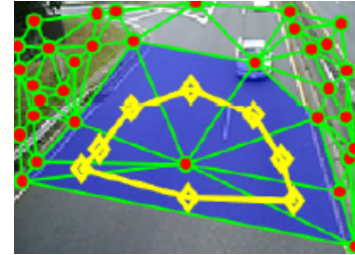

1.2: Traffic video frame vortex

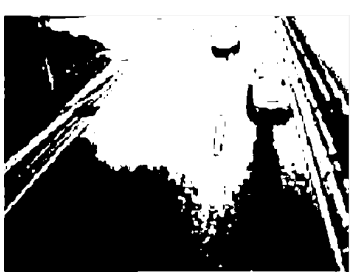

1.3: Frame binary image

FiguRE 1. Sample video frame barycentric vortex

A vortex is a collection of nesting, usually non-concentric, path-connected, barycentric, intersecting cycles. A cycle $E$ (denoted by cyc $E$ ) contains vertexes so that each pair of vertexes $p, q \in \operatorname{cyc} E$ in the cycle is path-connected, i.e., there is a sequences of edges leading from vertex $p$ to vertex $q$ in the cycle. The vertexes in barycentric cycles are the barycenters (intersection of the median lines) of triangles. An optical vortex is a vortex constructed from vertexes that are picture elements (pixels), snapshots of reflected light from surfaces recorded in a video frame (for a sample optical vortex, see Example 1).

Example 1. A barycentric optical vortex containing a single cycle (in yellow) is shown in the triangulation of the traffic video frame in Fig. 1.1 is shown in Fig. 1.2.

Vortex cycles are examples of nerve structures (called an optical vortex nerve). A vortex nerve is a collection of nesting, possibly overlapping filled vortexes attached to each other and have nonempty intersection [1, 19, 16, 15, 14]. A filled vortex has a boundary that is a simple closed curve and a nonempty interior. Due to the fact that the optical cycles intersect, i.e., the cycles in such vortexes have one or more common vertexes (see, e.g., Example 5). Because the paths between vertexes are on intersecting cycles in an optical vortex are bi-directional, we obtain the following result.

Theorem 1. $[17, \S 4.13$, p. 212] An optical vortex has a free Abelian group representation.

Recall that a group is a nonempty set $V$ (for vortex) equipped with a binary operation (represented here with a + (traverse or move)), so that each vertex $p$ of $V$ has an inverse $-p$ with $p+(-p)=0$ (i.e., no traversal or movement occurs) and $p+q=q+p$ (Abelian property). That is, traversing the edges from $p$ to $q$ in the vortex can always be followed by a traversal of the edges from $q$ to $p$, which takes us back to where we started. A zero move is the identity element of 
the group. For example, $p+0$ reads 'no traversal occurs at $p$ '. In a free Abelian group representation of a vortex nerve, each vertex in the nerve can be written as a summation of the generating elements. The number of generators in such a group is the rank of the group.

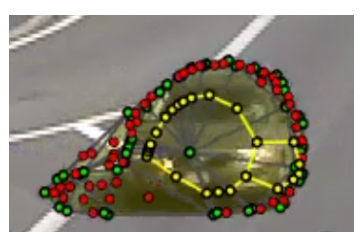

2.1: Traffic optical vortex nerve 1

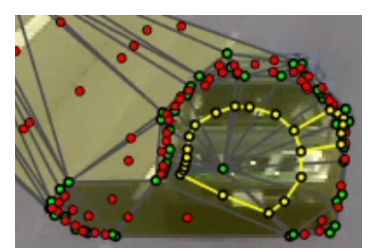

2.2: Traffic optical vortex nerve 2

Figure 2. Sample traffic video frame optical vortex nerves

Example 2. Sample traffic video frame optical vortex nerves are shown in Fig. 2. The vertexes on the edges attached between the inner yellow cycles are the generators of the free Abelian group representations of these nerves.

A Ghrist barcode, usually called a persistence barcode, is a topology-of-data pictograph that represents that appearance and disappearance of consecutive sequences of video frames having a particular feature value [8], [9, §5.13, pp. 104-106]. The origin of topology-of-data barcodes can be traced back to H. Edelsbrunner, D. Letscher and A. Zomorodian [6], [7]. For a complete view of the landscape for a topology-of-data barcode viewed as a multiset of intervals ${ }^{1}$, see J.A. Perea [13].

Example 3. An overview of the steps leading to a barcode for a traffic video is given in Fig. 3. These steps in the construction of an optical vortex nerve begin with the triangulation of a moving traffic video frame shape. Two measures of shape structure (Fermi energy and counts of the basic parts of each nerve) are reflected in varying length horizontal bars in a 2D barcode for each video. From shape energy and shape part counts, a $3 D$ barcode is constructed for each shape vortex nerve.

\section{Preliminaries}

This section briefly introduces Betti numbers and Fermi energy.

2.1. Betti numbers. There two forms of Betti numbers, an algebraic Betti number (number of generators of a free Abelian group, denote simply by $\beta$ ), introduced in J.R. Munkres [11, §1.4, p. 24] and three geometric Betti numbers that give us the cardinality of geometric structures in a CW complex formed by the triangulation of a video frame shape, introduced by A.J. Zomorodian $[24, \S 4.3 .2$, p. $55]$. The focus here is on geometric Betti numbers, which are more informative in characterizing triangulated video frame shapes. The important thing to notice is that we isolate and triangulate moving vehicle shapes in traffic video frames. That is, we restrict Betti numbers to triangulated shapes [20] as opposed to triangulation of an entire video frame.

\footnotetext{
${ }^{1}$ Many thanks to Vidit Nanda for pointing this out.
} 


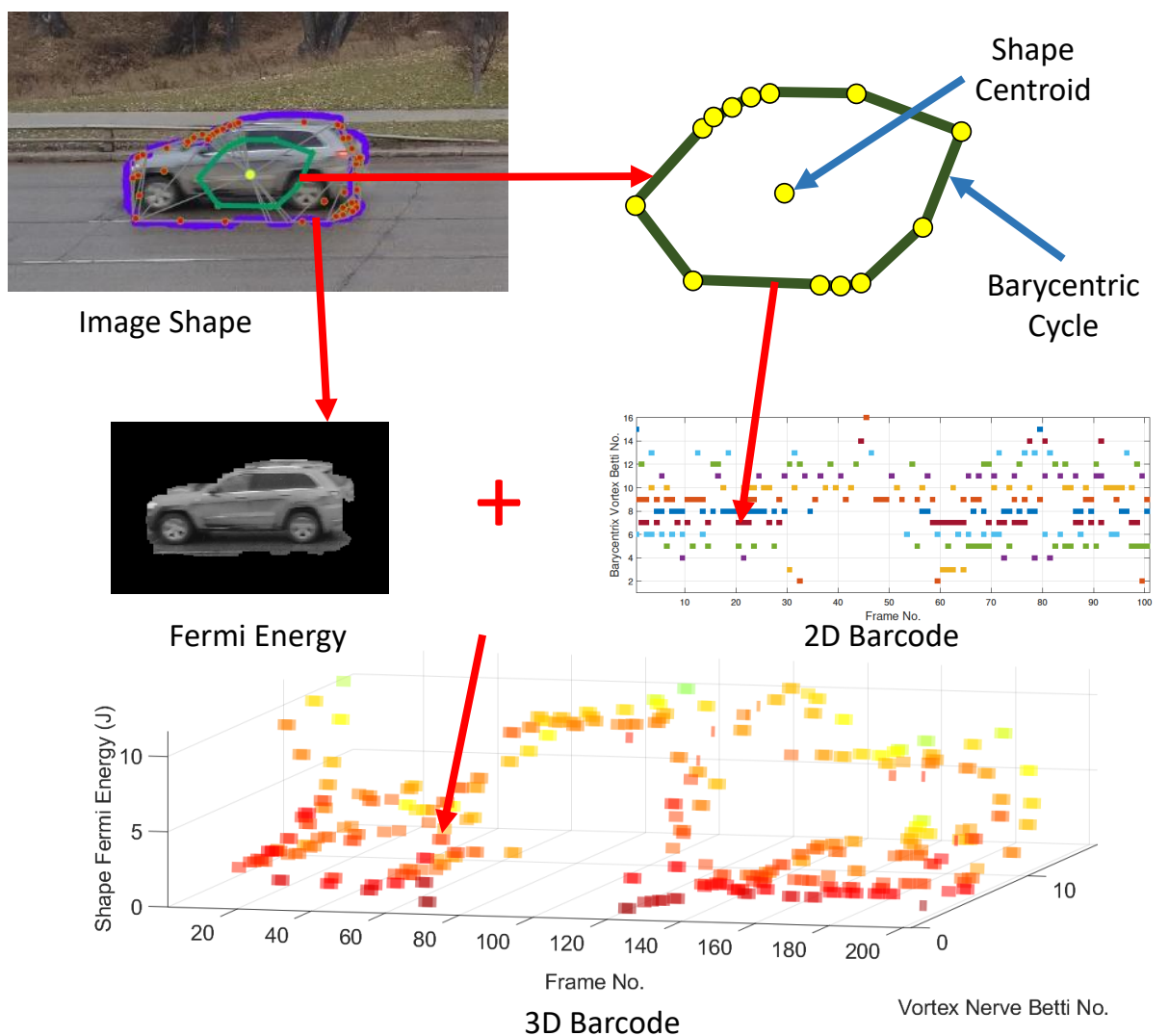

Figure 3. Barycentric Cycle.

In the context of finite $\mathrm{CW}$ complexes found on triangulated finite bounded planar regions, triangulated video frame shapes are characterized by the geometric Betti numbers, namely, $\mathcal{B}_{0}$ (cell or $\mathrm{CW}$ complex components count), $\mathcal{B}_{1}$ (vortex nerve cycle count) and $\mathcal{B}_{2}$ (hole or void count). By contrast, from an algebraic perspective, the Betti number of a vortex nerve is a count of the number of generating elements that define a free Abelian group representation of the nerve.

In triangulated traffic video frames, vertexes are the centroids of dark frame regions. Each frame dark region absorbs sunlight and hence the dark regions are considered holes with corresponding $\beta_{2}$ counts. In that case, $\beta_{0}$ counts the number of centroidal vertexes, edges and filled triangles in a video frame. And $\beta_{1}$ is a count of the number of barycentric cycles on a triangulated video frame.

Example 4. In Fig. 1.2, we have the following Betti numbers:

\section{Geometric views:}

$$
\begin{aligned}
& \beta_{0}=8+40(\text { filled triangle count })+34 \text { vertexes }+34 \text { edges }=116 . \\
& \left.\beta_{1}=1 \text { (cycle count }\right) . \\
& \left.\beta_{2}=75 \text { (hole count }\right) .
\end{aligned}
$$


Algebraic view:in Fig. 2.2.

$$
\begin{aligned}
& \beta=2 \text {, i.e., } 4 \text { generators, i.e., end vertices on the edges, } \\
& \text { connected between the yellow barycentric cycles. }
\end{aligned}
$$

In the binary image for Fig. 1.2 given in Fig. 1.3, there are a total of 150 bounded regions (75 of the bounded regions are holes).

In this paper, only geometric Betti numbers are used in tracking persistent structures in sequences of video frames.

2.2. Fermi energy. The Hummel form of Fermi Energy [10, §6, p. 69, Eqn. (6.11)] derived from the pixel population of an object shape is used in this paper.

$$
E_{F}=\frac{k}{2 m_{0}}\left(\frac{3 \pi^{2} N}{V}\right)^{2 / 3}
$$

where $N$ is the number of pixels in a moving object shape, $V$, total area of the moving objects and $m_{0}$, the average intensity of the object shape pixels (in greyscale). A scaling factor $k$ was used to scale the values appropriately. This form of Fermi energy for structures in digital images appears in S. Pradikar, J. Sil and A.D. Das [21, p. 807].

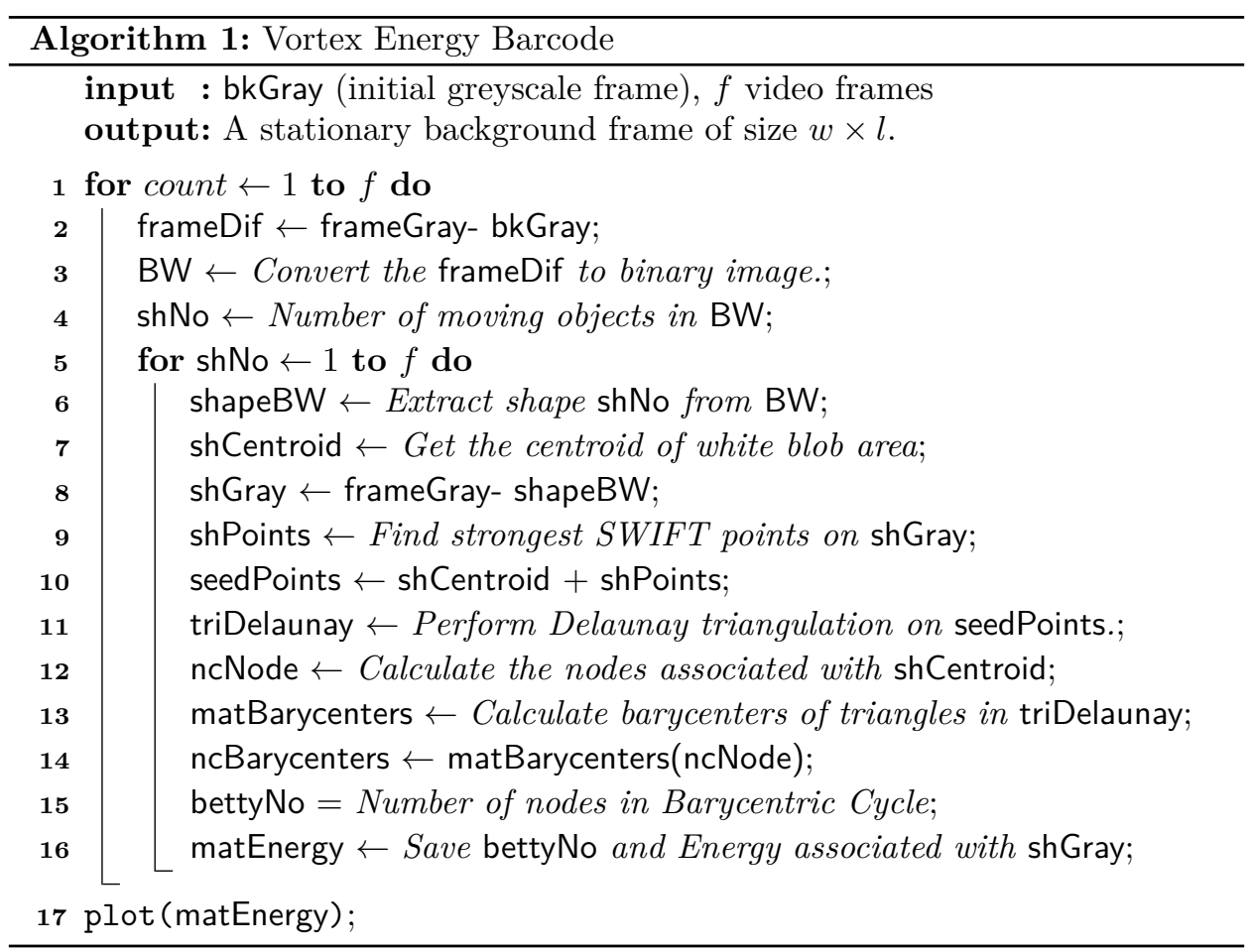

To isolate moving objects in each video frame, background subtraction was used, i.e., an initial video frame that displays no moving objects is subtracted from the remaining fames in the video. In effect, subtracting an initial frame from the 
remaining frames in a video makes it possible to remove all background (dark) regions in a video. Morphological operations were performed on the binary images to further isolate the object shapes in the remaining frames. Algorithm 1 gives the basic pseudo-code to construct optical vortex nerves on triangulated video frame shapes.

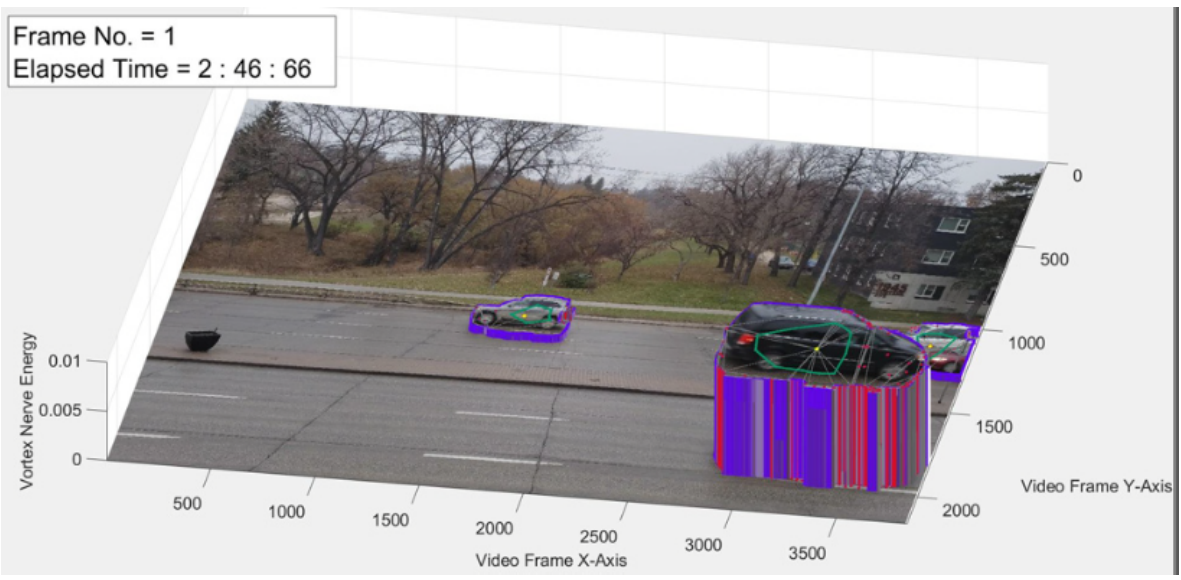

Figure 4. Shape Fermi Energy in traffic Video Frame

Visualization of shape pixel intensities is represented by a 3D projection of isolated frame object shapes.

Example 5. Shape Fermi energy in a video frames is shown in Fig. 4 for a traffic video of Portage Avenue in Winnipeg. From Alg. 1, Fermi energy results from extracting the number of pixels $N$ in a $3 D$ projection of a $2 D$ vehicle shape.

2.3. Time Complexity Analysis. Figure 5 shows the results of time complexity analysis for Alg. 1. In estimating the complexity of Alg. 1, both theoretical and actual time values were calculated. The time taken for the built-in functions (e.g., video frame extraction, video frame exporting, triangulation) were not considered, since these functions are optimized. When calculating the theoretical time complexity addition, subtraction, multiplication and division were considered as 4 different calculations.

The video frame shape triangulation method introduced in this paper has theoretical time complexity $O\left(m n^{3}\right)$, which is computed in terms of $m$ (pixel area of a moving object) and $n$ (number of moving objects) in a video frame. An experimentally derived scaling factor of $k$ is used to align each theatrical graph with the actual time graph. The value of $k$ is in the order of $1 \times 10^{-6}$ and varies slightly depending on each time curve shown in Fig.5.

It is evident from Fig. 5 that the theoretical and the actual time complexity curves follow each other very closely. This is especially the case as the number of object shapes and the object shape area increase. There are some deviations from this observation, when the object area and the number is small, which is to be expected. This anomaly results from not considering the impact of some functions, which are at work in the background of the system, which affects the time taken 


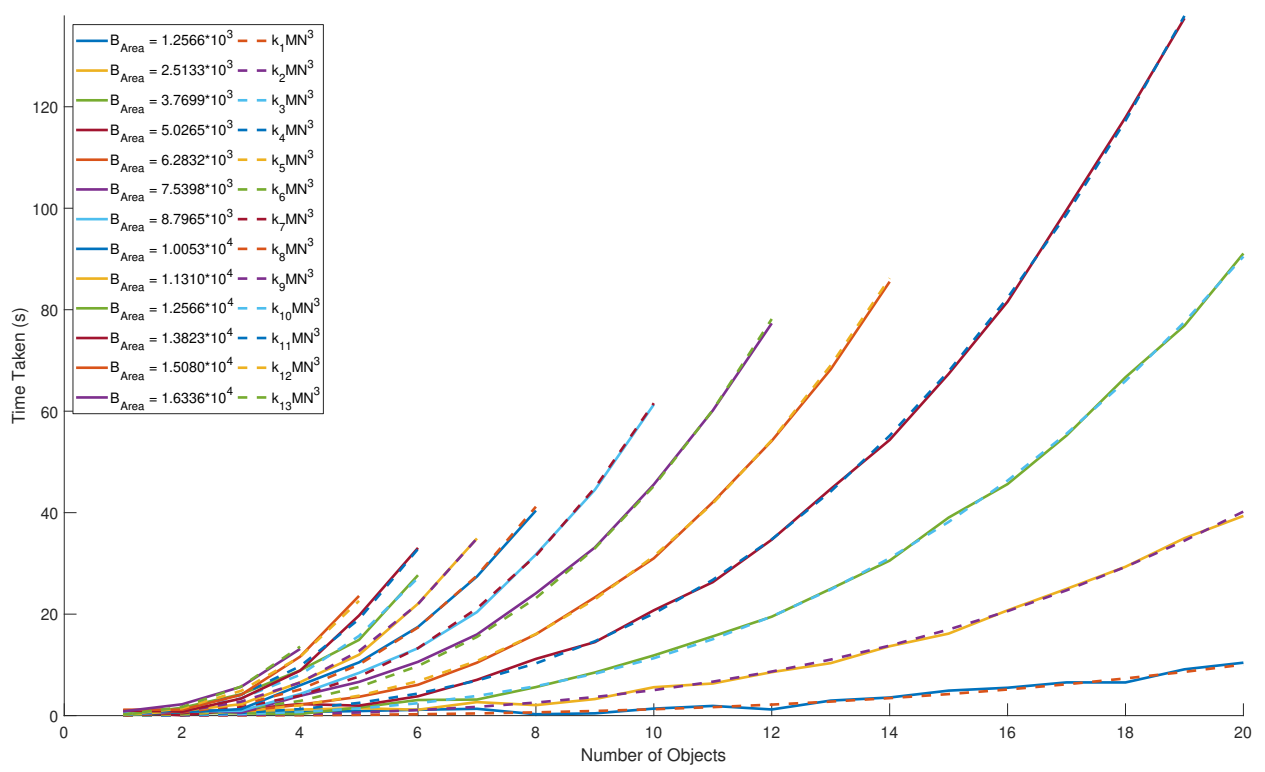

Figure 5. Time complexity.

for processing. But when the computation time increases, the influence of the background functions becomes minimal compared to the overall computation time.

\section{REsults}

Two of the results of this research reflect the influence of increasing shapes in sequences of video frames (see [3]).

Lemma 1. Let shA, shA' be object shapes in a pair of adjacent video frames with sh $A^{\prime}$ appearing after shA. Also, let $N, V, E, N^{\prime}, V^{\prime}, E^{\prime}$ be the number of shape pixels, shape area, and Fermi energy in shA, sh $A^{\prime}$, respectively. Then $N^{\prime}>V^{\prime}, N^{\prime}>N$ and $N>V$ implies $E^{\prime}>E$.

Proof. From Eqn. 2.2, we obtain the desired result.

Observe that nesting, usually non-concentric cycles covering all or the principal part of the interior of a video frame shape, form a ribbon. A ribbon is a collection of such nesting filled cycles, which is an example of a vortex nerve. A vortex nerve is a collection of nesting cycles that have non-empty intersection. This yields the following result.

Theorem 2. [18].

$A$ ribbon is a vortex nerve.

Theorem 3. Let shA, sh $A^{\prime}$ be object shapes covered by nesting, non-concentric filled cycles in a pair of adjacent video frames with sh $A^{\prime}$ appearing after shA. Let $\beta_{1}$ be the cycle count Betti number for shA, $\beta_{1}^{\prime}$ for sh $A^{\prime}$. Assume that shape sh $A^{\prime}$ has $N_{\text {pixels }}^{\prime}>V_{\text {area }}^{\prime}$ and shA has $N_{\text {pixels }}>V_{\text {area }}$. Then $\beta_{1}^{\prime}>\beta_{1}$ implies $E^{\prime}>E$.

Proof. From Theorem $2, \operatorname{sh} A, \operatorname{sh} A^{\prime}$ are vortex nerves. $\beta_{1}^{\prime}>\beta_{1}$ indicates that the number of nesting, non-concentric cycles in $\operatorname{sh} A^{\prime}$ is greater than the number of 
cycles in $\operatorname{sh} A$. The outer cycle (call it $\operatorname{cyc} A^{\prime}$ ) on the interior of $\operatorname{sh} A^{\prime}$ has the remaining cycles of $\operatorname{sh} A^{\prime}$ nested inside cyc $A^{\prime}$. Also, from $\beta_{1}^{\prime}>\beta_{1}$, the number of pixels $N^{\prime}>V^{\prime}$ in the interior of cyc $A^{\prime}$ is greater than the number of pixels $N>V$ in the interior of $\operatorname{cyc} A$. Hence, from Lemma $1, E^{\prime}>E$.

Barcoding traffic videos provides a concise means of tracking the persistences of shapes across sequences of video frames. Each occurrence of a frame shape is represented by a horizontal bar in 2D Ghrist pictographs called barcodes, introduced by $\mathrm{R}$. Ghrist $[8,9]$ and others $[7,6,13,4]$ and later extended to 3D persistence barcodes in [5].

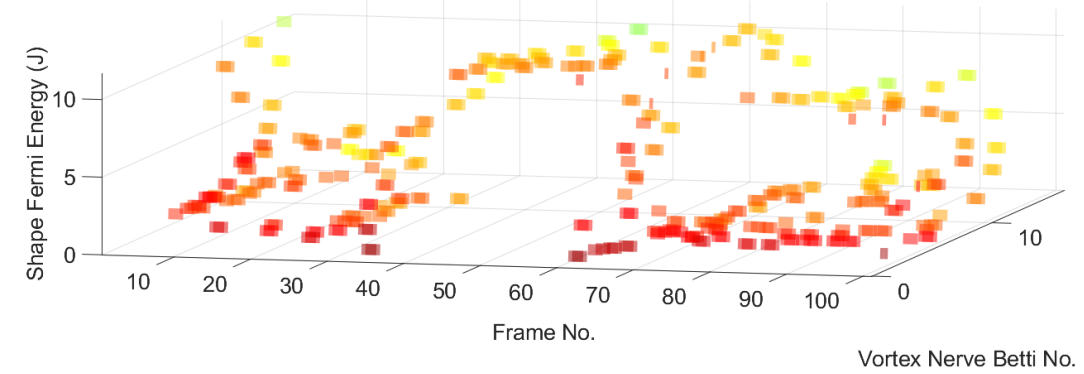

Figure 6. Example 3D Barcode for Traffic Video

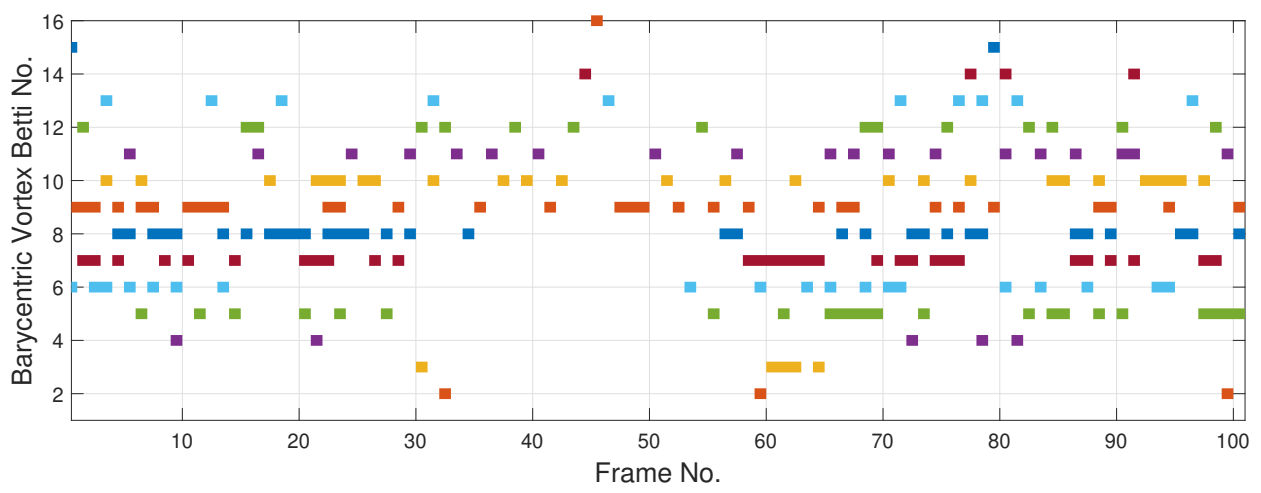

Figure 7. Example Barcode for Traffic Video

The basis for a picture proof of Theorem 3 is given in the 3D pesistence barcode in Example 6.

Example 6. In the 3D persistence barcode in Fig. 4, the cycles Betti number $\beta_{1}$ is also vortex Betti number. Uniformly in Fig. 4, as the cycles Betti number $\beta_{1}$ increases, there is a corresponding increase in shape Fermi energy across a sequence of frames. This tends to corrobarate the observation in Theorem 3.

A 2D persistence barcode structured in terms of barycentric cycle counts in the vortex Betti number $\beta_{1}$, exhibits which of the $\beta_{1}$ vortex cycle counts tend to be 
more persistent over a sequence of vehicular traffic video frames. A barycentric cycle is a simple, closed curve in which its vertexes are triangle barycenters that are path-connected.

Example 7. In the 2D persistence barcode given in Fig. 7, the vortex cycles Betti number $\beta_{1}$ ranges from 2 to 16 over a sequence of traffic video frames. The midrange $\beta_{1}$ counts (from 7 to 9) have frequent extended horizontal bars spanning a sequence of frames with extended bars ranging over 2 to 7 consecutive frames. In other words, shapes with mid-range $\beta_{1}$ counts tend to be more persistent.

\section{REFERENCES}

1. M.Z. Ahmad and J.F. Peters, Maximal centroidal vortices in triangulations. a descriptive proximity framework in analyzing object shapes, Theory and Applications of Math. \& Comp. Sci. 8 (2018), no. 1, 38-59, ISSN 2067-6202.

2. P. Alexandroff and H. Hopf, Topologie. Band i, Springer, Berlin, 1935, Zbl 13, 79; reprinted Chelsea Publishing Co., Bronx, N. Y., 1972. iii+637 pp., MR0345087.

3. A.P.H. Don and J.F. Peters, Ci lab traffic video archive, https://drive.google.com/drive/ folders/1_sRajgxwy3TgVOFpqe62N29fZsUAlOkH?usp=sharing.

4. —_ Ghrist barcoded video frames. Application in detecting persistent visual scene surface shapes captured in videos, Quaestiones Mathematicae 37 (2014), no. 2, 249-263, Zbl 1397.54037,Zbl 1397.54038.

5. A.P.H. Don, J.F. Peters, S. Ramanna, and A. Tozzi, Topological inference from spontaneous activity structures in fmri videos with peristence barcodes, biRxiv 1101 (2019), no. 809293, 1-11, https://doi.org/10.1101/809293.

6. H. Edelsbrunner, D. Letscher, and A. Zomorodian, Topological persistence and simplification, 41st Annual Symposium on Foundations of Computer Science, IEEE Comput. Soc. Press, Los Alamitos, California, 2000, MR1931842, pp. 454-463.

7. 511-533, MR1949898.

8. R. Ghrist, Barcodes: the persistent topology of data, Bull. Amer. Math. Soc. (N.S.) 45 (2008), no. 1, 61-75, MR2358377.

9. R.W. Ghrist, Elementary applied topology, University of Pennsylvania, 2014, vi+269 pp. ISBN: 978-1-5028-8085-7.

10. R.E. Hummel, Electronic properties of materials, 4th ed., Springer, New York, U.S.A., 2011, viii+488 pp., 539 pp. ISBN: ISBN 978-1-4419-8163-9

11. J.R. Munkres, Elements of algebraic topology, $2^{\text {nd }}$ ed., Perseus Publishing, Cambridge, MA, 1984, ix + 484 pp., ISBN: 0-201-04586-9, MR0755006.

12. S.A. Naimpally and J.F. Peters, Topology with applications. topological spaces via near and far, World Scientific, Singapore, 2013, xv + 277 pp, Amer. Math. Soc. MR3075111.

13. J.A. Perea, A brief history of persistence, arXiv 1809 (2018), no. 036249, 1-11.

14. J.F. Peters, Proximal planar shape signatures. Homology nerves and descriptive proximity, Advan. in Math: Sci. J 6 (2017), no. 2, 71-85, Zbl 06855051.

15. __ Proximal planar shapes. correspondence between triangulated shapes and nerve complexes, Bulletin of the Allahabad Mathematical Society 33 (2018), 113-137, MR3793556, Zbl 06937935, Review by D. Leseberg (Berlin).

16. _ Proximal vortex cycles and vortex nerve structures. non-concentric, nesting, possibly overlapping homology cell complexes, Journal of Mathematical Sciences and Modelling 1 (2018), no. 2, 56-72, ISSN 2636-8692, www.dergipark.gov.tr/jmsm, See, also, https://arxiv.org/abs/1805.03998.

17. Computational geometry, topology and physics of digital images with applications. Shape complexes, optical vortex nerves and proximities, Springer Nature, Cham, Switzerlan, 2020, xxv+440 pp.,ISBN 978-3-030-22191-1/hbk; 978-3-030-22192-8/ebook, Zbl07098311.

18. _ Planar ribbon complexes and their approximate proximities. Ribbon nerves, betti numbers and planar divisions, Bulletin of the Allahabad Mathematical Society (2020), 1-14, to appear. See, also, arXiv,https://arXiv:1911.09014. 
19. J.F. Peters and S. Ramanna, Shape descriptions and classes of shapes. A proximal physical geometry approach, Advances in feature selection for data and pattern recognition (B. Zielosko U. Sta?czyk and L.C. Jain, eds.), Springer, 2018, MR3811252, pp. 203-225.

20. __ Shape descriptions and classes of shapes. a proximal physical geometry approach, Advances in Feature Selection for Data and Pattern Recognition, ISRL 138, Springer, 2018, https://doi.org/10.1007/978-3-319-67588-6, MR3811252, pp. 203-225.

21. S. Pradikar, J. Sil, and A.D. Das, Region identification of infected rice images using the concept of fermi energy, Advances in Computing \& Inform. Technology (N. Meghanathan et al., ed.), Springer-Verlag, Berlin, 2013, pp. 805-811.

22. J.H.C. Whitehead, Simplicial spaces, nuclei and m-groups, Proceedings of the London Math. Soc. 45 (1939), 243-327.

23. Combinatorial homotopy. I, Bulletin of the American Mathematical Society 55 (1949), no. 3, 213-245, Part 1.

24. A.J. Zomorodian, Computing and comprehending topology persistence and hierarchical morse complexes, Ph.D. thesis, University of Illinois at Urbana-Champaign, Graduate College, 2001, supervisor: H. Edelsbrunner, 199pp.

Computational Intelligence Laboratory, University of Manitoba, WPG, MB, R3T 5V6, CANADA

Email address: pilippua@myumanitoba.ca

Computational Intelligence Laboratory, University of Manitoba, WPG, MB, R3T 5V6, Canada and Department of Mathematics, Faculty of Arts and Sciences, Adiyaman University, 02040 Adiyaman, Turkey

Email address: James.Peters3@umanitoba.ca

Applied Computer Science Department, University of Winnipeg, WPG, MB, R3B 2E9, CANADA

Email address: s.ramanna@uwinnipeg.ca 